\title{
Finding untriggered gamma-ray transients in the Fermi GBM data
}

\section{M. Hui*}

NASA Marshall Space Flight Center

E-mail: c.m.hui@nasa.gov

\section{S. Briggs}

University of Alabama in Huntsville

E-mail: briggsms cuah. edu

\section{P. Veres}

University of Alabama in Huntsville

E-mail: pv0004@uah. edu

\section{R. Hamburg}

University of Alabama in Huntsville

E-mail: rkh0007@uah.edu

The all-sky monitoring capability of Fermi GBM makes it ideal for finding transients. Fermi GBM triggers on events such as gamma-ray bursts, soft gamma-ray repeaters, X-ray bursters, solar flares, and terrestrial gamma-ray flashes. A previous systematic search in offline data for Xray bursts has uncovered untriggered gamma-ray bursts, and currently there is a dedicated offline search pipeline looking for weak transients undetected by the onboard trigger conditions. The untargeted search looks for significant signals in two or more detectors at various timescales in the continuous background-subtracted data. It detects $\sim 80$ short GRB candidates per year in addition to the $\sim 40$ short GRBs that trigger onboard. Since July 2017, these candidates are published by the GRB Coordinates Network as Fermi-GBM Subthreshold Notices, accompanied by localization maps and lightcurves. At present only short GRB candidates are published, expansion to long GRBs and other transient types are forthcoming.

7th Fermi Symposium 2017

15-20 October 2017

Garmisch-Partenkirchen, Germany

\footnotetext{
*Speaker.
} 
Since launching in 2008, the Gamma-ray Burst Monitor (GBM) onboard the Fermi Gammaray Space Telescope has detected over 2000 gamma-ray bursts (GRBs), $\sim 2$ every 3 days. Each year, GBM detects $\sim 200$ long GRBs and $\sim 40$ short GRBs. To further our work on multiwavelength and multi-messenger follow up, we have developed additional algorithms to increase the detection rate of GRBs in the GBM data.

Similar to the search algorithm used by the GBM flight software, the untargeted search looks for excess signals in 2 GBM NaI detectors. However, an improved background model is used and the thresholds are $2.5 \sigma$ and $1.25 \sigma$ above background, rather than the $4.5 \sigma$ used by the flight software. Additionally the 2 detectors must have valid geometry for a point source. The search goes through 18 timescales from 64ms to 32s, and 4 energy ranges that are optimized for short GRBs, by binning the continuous time-tagged event data, which has $2 \mu$ s resolution and 128 energy channels. A one-day Poisson probability is calculated for each event and a threshold for notice is established at 1e-5. GCN notices for these subthreshold event is available at https://gcn.gsfc.nasa.gov/fermi_gbm_subthreshold.html. For archival candidates before April 2017 and back to 2013, these are available at our website http://gammaray.nsstc.nasa.gov/gbm/science/sgrb_search.html. Localization FITS file, contour sky map, and the lightcurve are available with the GCN notices. The time delay for the notice publication will range from 0.5 to 6 hours, due to telemetry schedule. The expected rate of notice will be $\sim 70$ per month, during periods of galactic transients activity the rate is expected to be higher.

Verification with other instruments such as Swift and INTEGRAL are ongoing. Figure 1 shows the lightcurve of candidate 423745096 which corresponds to GRB 140606A detected by Swift [1]. This GRB did not trigger GBM onboard due to low peak flux. The GBM event is found at the same time as the Swift Burst Alert Telescope trigger time with consistent localization.

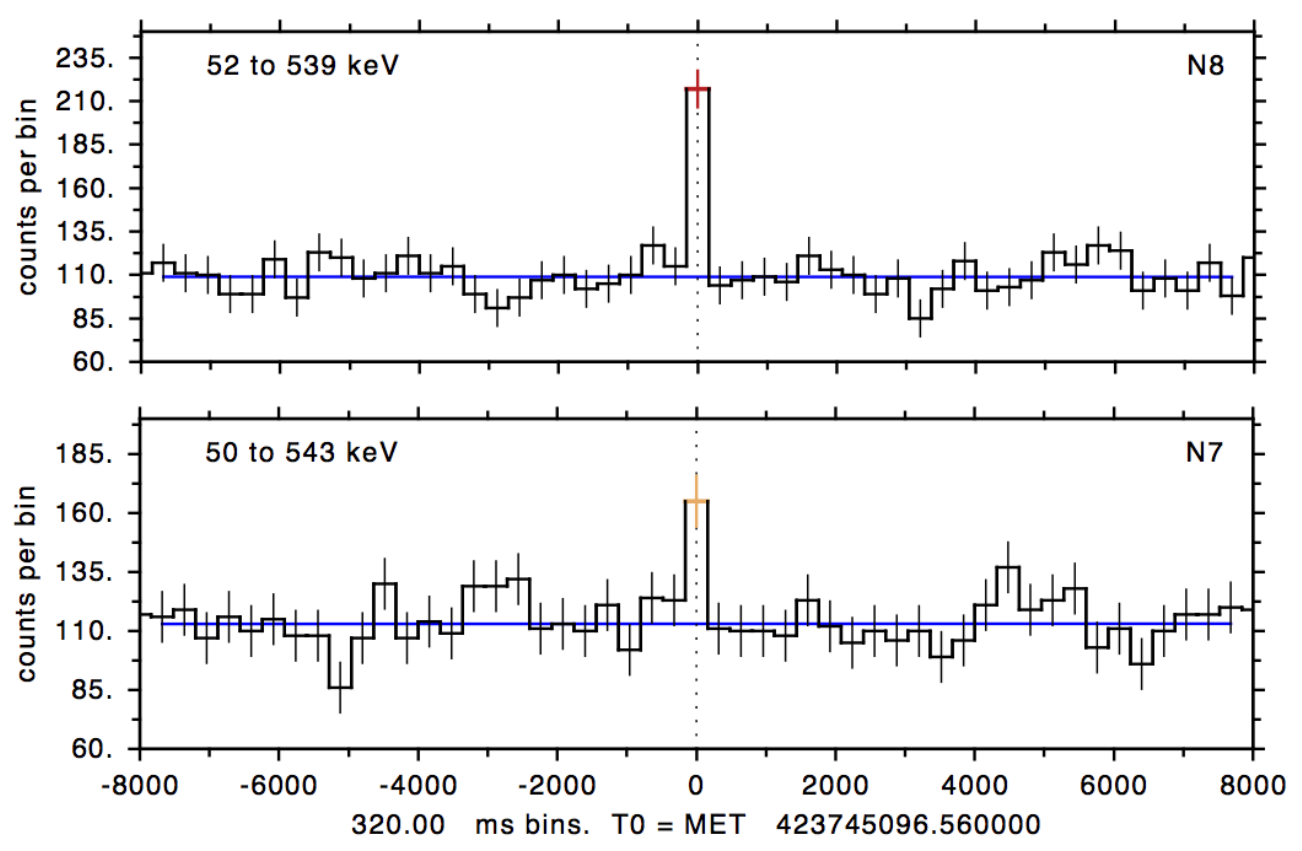

Figure 1: Lightcurves of the 2 brightest detectors for GRB 140606A. 
Figure 2 shows the transient candidate 527647422 which is a high reliability candidate with $>4 \sigma$ excess in 3 detectors and is found in 2.8 seconds timescale. This event is also found by Insight-HXMT [2] at $12 \sigma$ at $80-800 \mathrm{keV}$ during their commissioning phase and now named GRB 170921C.
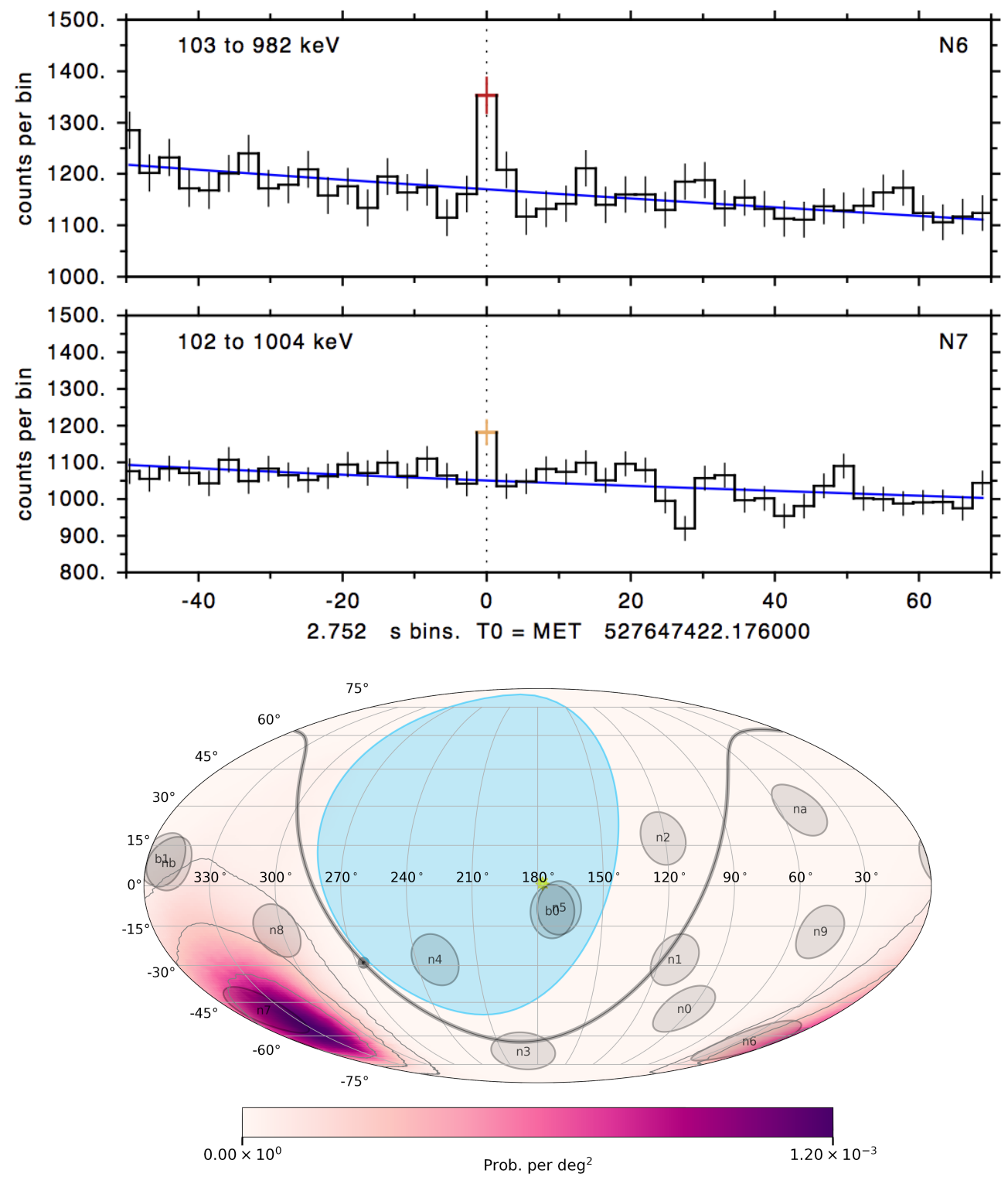

Figure 2: Top: Lightcurves of the 2 brightest detectors. Bottom: Localization map for Fermi-GBM 527647422, now identified as GRB 170921C. The grey line shows the Galactic plane, with the Galactic Center represented by the grey dot. The blue shaded region is occulted by the Earth. Each detector pointing is shown in grey disks. 


\section{References}

[1] M. C. Stroh, A. P. Beardmore, J. R. Cummings, et al., GRB Coordinates Network, Circular Service, No. 16353 (2014)

[2] Y. F. Zhang, S. L. Xiong, J. Y. Liao, et al., GRB Coordinates Network, Circular Service, No. 21919 (2017) 